\title{
UNIFIED GLOBAL OPTIMALITY CONDITIONS FOR SMOOTH MINIMIZATION PROBLEMS WITH MIXED VARIABLES *
}

\author{
VAIthilingam JeyakumaR $^{1}$, Sivakolundu SRISATKunarajah ${ }^{1}$ \\ AND NGUYen QuANG HuY ${ }^{1,2}$
}

\begin{abstract}
In this paper we establish necessary as well as sufficient conditions for a given feasible point to be a global minimizer of smooth minimization problems with mixed variables. These problems, for instance, cover box constrained smooth minimization problems and bivalent optimization problems. In particular, our results provide necessary global optimality conditions for difference convex minimization problems, whereas our sufficient conditions give easily verifiable conditions for global optimality of various classes of nonconvex minimization problems, including the class of difference of convex and quadratic minimization problems. We discuss numerical examples to illustrate the optimality conditions
\end{abstract}

Keywords. Nonconvex optimization, global optimization, optimality conditions, discrete constraints, box constraints, difference of convex functions, quadratic minimization.

Mathematics Subject Classification. 90C30, 90C45.

\footnotetext{
Received January 31, 2007. Accepted December 4, 2007.

* The authors are grateful to the referees for thier valuable suggestions and useful comments which have contributed to the final preparation of the paper. Research was partially supported by a grant from the Australian Research Council.

1 Department of Applied Mathematics, University of New South Wales, Sydney 2052, Australia; $\{$ jeya, sri\} @maths.unsw.edu.au

2 Hanoi Pedagogical University No. 2, Vinh Phuc, Vietnam.
} 


\section{INTRODUCTION}

In this paper, we consider the following smooth minimization problem with mixed variables.

$$
\begin{array}{cl}
\min _{x \in \mathbb{R}^{n}} & f(x) \\
\text { s.t. } & x_{i} \in\left[u_{i}, v_{i}\right], i \in I, \\
& x_{i} \in\left\{u_{i}, v_{i}\right\}, i \in J, \\
& I \cap J=\phi, I \cup J=\{1,2, \ldots, n\},
\end{array}
$$

where $u_{i}, v_{i} \in \mathbb{R}, u_{i}<v_{i}$ and $f$ is a twice continuously differentiable function on an open set containing the feasible set of $(M P)$. Model problems of the form $(M P)$ appear in numerous application areas, including electronic circuit design [10], computational chemistry $[4,11]$ and combinatorial optimization $[2,12]$, and cover, for instance, optimization problems with binary constraints [1], where $u_{i}=$ $0, v_{i}=1$ for all $i \in J$ and $I=\emptyset$, and box-constrained smooth optimization problems [3,14], where $J=\emptyset$.

Recently, global optimality conditions for various classes of problems $(M P)$ have been established separately for the box constrained problems, where $J=\emptyset$ $[1,3,9]$, and for problems with discrete constraints, where $I=\emptyset[13,15]$. Sufficient optimality conditions were established in [8] for quadratic minimization problems with box constraints. More recently, in [7] global optimality conditions were given separately for the minimization of difference of quadratic and convex functions constrained over box as well as over binary constraints. In [6], corresponding results were also given for smooth minimization problems. By considering $(M P)$, in this paper, we not only present unified global optimality conditions for these classes of problems extending and improving the corresponding recent results (see $[1,3,6-8])$ but also we provide optimality conditions for problems with mixed variables. Moreover, our extended necessary global optimality conditions now cover difference of convex minimization problems, whereas our sufficient conditions provide easily verifiable conditions for global optimality of various classes of nonconvex problems. Our approach is based on a minimizing quadratic underestimator, which is an underestimator at a feasible point (see [5-7]) at which it attains its global minimizer. Thus, a minimizing underestimator at a feasible point yields global optimality of the problem.

The outline of the paper is as follows. Section 2 presents necessary optimality conditions for various classes of problems $(M P)$, including difference of convex minimization problems. Section 3 provides conditions that are sufficient for global optimality of $(M P)$, and includes conditions for constructing a minimizing quadratic underestimation of a smooth function. 


\section{NeCESSARY GLOBAL CONDItions}

In this section we derive necessary conditions for a given feasible point to be a global minimizer of the optimization model problem $(M P)$. We then give several classes of optimization problems where the conditions can easily be checked.

We begin by giving the notations and definitions used throughout the paper. For a matrix, $A \succeq 0$ means that $A$ is positive semi-definite. A diagonal matrix with diagonal elements $a_{1}, a_{2}, \ldots, a_{n}$ is denoted by $\operatorname{diag}(A):=\operatorname{diag}\left(a_{1}, \ldots, a_{n}\right)$. For $f: \mathbb{R}^{n} \rightarrow \mathbb{R}$, the gradient and the Hessian of $f$ at $\bar{x}$ are denoted by $\nabla f(\bar{x})$ and $\nabla^{2} f(\bar{x})$, respectively. Clearly, for each $x \in \mathbb{R}^{n}, \nabla^{2} f(x) \in S^{n}$, where $S^{n}$ the space of all $(n \times n)$ symmetric matrices. The set of all positive semidefinite $n \times n$ symmetric matrices is denoted by $S_{+}^{n}$.

For the problem $(M P)$, put

$$
D=\left\{\left(x_{1}, \ldots, x_{n}\right)^{T} \in \mathbb{R}^{n} \mid x_{i} \in\left[u_{i}, v_{i}\right], \quad i \in I, \quad x_{i} \in\left\{u_{i}, v_{i}\right\}, \quad i \in J\right\} .
$$

Let $\bar{x}=\left(\bar{x}_{1}, \ldots, \bar{x}_{n}\right) \in D$. For $i=1,2, \ldots n$, define

$$
\tilde{\chi}_{i}= \begin{cases}-1 & \text { if } \bar{x}_{i}=u_{i} \\ +1 & \text { if } \bar{x}_{i}=v_{i} \\ (\nabla f(\bar{x}))_{i} & \text { if } \bar{x}_{i} \in\left(u_{i}, v_{i}\right)\end{cases}
$$

For $c_{i} \in \mathbb{R}, i=1,2 \ldots n$, define

$$
\tilde{c}_{i}= \begin{cases}\max \left\{0,-c_{i}\right\} & \text { if } i \in I \\ -c_{i} & \text { if } i \in J\end{cases}
$$

Define

$$
\tilde{D}:=\left\{\left(x_{1}, \ldots, x_{n}\right)^{T} \in \mathbb{R}^{n} \mid x_{i} \in\left[u_{i}, v_{i}\right], i \in I \cup J\right\}
$$

We now derive necessary conditions for global optimality.

Theorem 2.1. For $(M P)$, suppose that

$$
\max \left\{\partial^{2} f(z) / \partial x_{i}^{2} \mid z \in \tilde{D}\right\} \leq c_{i}
$$

for some $c_{i} \in \mathbb{R}, i=1,2, \ldots n$. If $\bar{x} \in D$ is a global minimizer of $(M P)$ then, for each $i=1,2, \ldots, n$,

$$
[N C] \quad \frac{1}{2} \tilde{c}_{i}\left(v_{i}-u_{i}\right)+\tilde{\chi}_{i}(\nabla f(\bar{x}))_{i} \leq 0 .
$$


Proof. Let $\bar{x}$ be a global minimizer of $(M P)$. Then, for every $x \in D$ there exists $z \in \tilde{D}$ such that

$$
\begin{aligned}
f(x)-f(\bar{x}) & =\frac{1}{2}\left(\sum_{j=1}^{n} \sum_{i=1}^{n}\left(\frac{\partial^{2} f(z)}{\partial x_{i} \partial x_{j}}\left(x_{i}-\bar{x}_{i}\right)\left(x_{j}-\bar{x}_{j}\right)\right)+\sum_{i=1}^{n}(\nabla f(\bar{x}))_{i}\left(x_{i}-\bar{x}_{i}\right)\right) \\
& =\frac{1}{2} \sum_{i=1}^{n}\left(\sum_{j=1}^{n} \frac{\partial^{2} f(z)}{\partial x_{i} \partial x_{j}}\left(x_{i}-\bar{x}_{i}\right)\left(x_{j}-\bar{x}_{j}\right)+(\nabla f(\bar{x}))_{i}\left(x_{i}-\bar{x}_{i}\right)\right) .
\end{aligned}
$$

Since $\bar{x}$ is a global minimizer of $(M P)$, for each $x \in D$,

$$
\sum_{i=1}^{n}\left(\sum_{j=1}^{n} \frac{1}{2} \frac{\partial^{2} f(z)}{\partial x_{i} \partial x_{j}}\left(x_{i}-\bar{x}_{i}\right)\left(x_{j}-\bar{x}_{j}\right)+(\nabla f(\bar{x}))_{i}\left(x_{i}-\bar{x}_{i}\right)\right) \geq 0 .
$$

Then, for each $i=1,2, \ldots, n$,

$$
\frac{1}{2} c_{i}\left(x_{i}-\bar{x}_{i}\right)^{2}+(\nabla f(\bar{x}))_{i}\left(x_{i}-\bar{x}_{i}\right) \geq 0,\left(x_{1}, x_{2}, \ldots x_{n}\right)^{T} \in D .
$$

Otherwise, there exist $i_{0}$ and $x_{i_{0}}$ such that (5) is not fulfilled. Then, by taking $\tilde{x}=\left(\tilde{x}_{1}, \ldots, \tilde{x}_{n}\right)$ such that $\tilde{x}_{i}=\bar{x}_{i}, i \neq i_{0}$ and $\tilde{x}_{i_{0}}=x_{i_{0}}$, one finds $\tilde{z} \in \tilde{D}$ satisfying

$$
\begin{aligned}
f(\tilde{x})-f(\bar{x}) & =\frac{1}{2}\left(\frac{\partial^{2} f(\tilde{z})}{\partial x_{i_{0}}^{2}}\right)\left(\tilde{x}_{i_{0}}-\bar{x}_{i_{0}}\right)^{2}+(\nabla f(\bar{x}))_{i_{0}}\left(\tilde{x}_{i_{0}}-\bar{x}_{i_{0}}\right) \\
& \leq \frac{1}{2} c_{i_{0}}\left(\tilde{x}_{i_{0}}-\bar{x}_{i_{0}}\right)^{2}+(\nabla f(\bar{x}))_{i_{0}}\left(\tilde{x}_{i_{0}}-\bar{x}_{i_{0}}\right) \\
& <0
\end{aligned}
$$

which contradicts (4).

We now show that (5) is equivalent to $[N C]$ by considering the following three cases.

Case 1. $\bar{x}_{i}=u_{i}$. If $i \in I$ then (5) holds if and only if

$$
\frac{1}{2} c_{i}\left(x_{i}-u_{i}\right)+(\nabla f(\bar{x}))_{i} \geq 0, \forall x_{i} \in\left(u_{i}, v_{i}\right] .
$$

If $c_{i} \geq 0$ then (6) holds if and only if $(\nabla f(\bar{x}))_{i} \geq 0$. Indeed, if $(\nabla f(\bar{x}))_{i}<0$, by taking $x_{i}$ sufficiently close to $u_{i}$, we obtain $\frac{1}{2} c_{i}\left(x_{i}-u_{i}\right)+(\nabla f(\bar{x}))_{i}<0$. This contradicts (6). Conversely, if (6) holds then (5) trivially holds.

If $c_{i}<0$ then we clearly see that (6) holds if and only if

$$
\frac{1}{2} c_{i}\left(v_{i}-u_{i}\right)+(\nabla f(\bar{x}))_{i} \geq 0 .
$$


If $i \in J$ then (5) holds if and only if

$$
\frac{1}{2} c_{i}\left(x_{i}-u_{i}\right)+(\nabla f(\bar{x}))_{i} \geq 0, \forall x_{i} \neq u_{i}
$$

i.e., (5) holds if and only if

$$
\frac{1}{2} c_{i}\left(v_{i}-u_{i}\right)+(\nabla f(\bar{x}))_{i} \geq 0
$$

Therefore $[\mathrm{NC}]$ holds.

Case 2. $\bar{x}_{i}=v_{i}$. The equivalence of (5) and $[N C]$ follows by similar arquments as in Case 1 above.

Case 3. $\bar{x}_{i} \in\left(u_{i}, v_{i}\right)$. Then (5) holds if and only if $(\nabla f(\bar{x}))_{i}=0$ and $c_{i} \geq 0$.

Indeed, if $\left(\nabla f\left(\overline{<} \bar{x}_{i}, x\right)\right)_{i}>0$ then, by taking $x_{i}$ sufficiently close to $\bar{x}_{i}$ and $x_{i}$ we have

$$
\left(x_{i}-\bar{x}_{i}\right)\left[\frac{1}{2} c_{i}\left(x_{i}-\bar{x}_{i}\right)+(\nabla f(\bar{x}))_{i}\right]<0
$$

which contradicts (5). On the other hand if $(\nabla f(\bar{x}))_{i}=0$ and $c_{i} \geq 0$ then obviously (5) holds, and so does $[N C]$.

Combining the above three cases, we obtain the conclusion.

Note from Theorem 2.1 that if $\bar{x} \in D$ is a global minimizer of $(M P)$ then, for each $i \in I, \tilde{\chi}_{i}(\nabla f(\bar{x}))_{i} \leq 0$, which is a necessary condition for $\bar{x}$ to be a local minimizer of $f$ over the box $\prod_{i \in I}\left[u_{i}, v_{i}\right]$.

Example 2.1. Consider the following problem

$$
\min _{x \in \mathbb{R}^{2}}\left\{x_{1}^{4}+x_{1}^{2}-x_{2}^{2}+x_{2} \mid x_{1} \in[-1,1], x_{2} \in\{-1,1\}\right\} .
$$

Let $f(x)=x_{1}^{4}+x_{1}^{2}-x_{2}^{2}+x_{2}$. Then $\nabla f(x)=\left(4 x_{1}^{3}+2 x_{1},-2 x_{2}+1\right)^{T}$.

$$
\begin{aligned}
& \frac{\partial^{2} f(z)}{\partial x_{1}^{2}}=12 z_{1}^{2}+2 \leq 14, \text { for each } z_{1}, z_{2} \in[-1,1] \\
& \frac{\partial^{2} f(z)}{\partial x_{2}^{2}}=-2, \quad \text { for each } z_{1}, z_{2} \in[-1,1]
\end{aligned}
$$

The points $\bar{x}=(0,1)$ and $\bar{y}=(0,-1)$ are local minimizers of the problem. Take $c_{1}=14, c_{2}=-2$. Then it is easy to check that the necessary condition $[N C]$ does not hold at the local minimizer $\bar{x}=\left(\bar{x}_{1}, \bar{x}_{2}\right)=(0,1)$ because

$$
\tilde{c}_{1}+\tilde{\chi}_{1}(\nabla f(\bar{x}))_{1}=0 \text { and } \tilde{c}_{2}+\tilde{\chi}_{2}(\nabla f(\bar{x}))_{2}=1>0 .
$$

Thus $\bar{x}=(0,1)$ is not a global minimizer of $f$. On the other hand, we can check that

$$
\tilde{c}_{1}+\tilde{\chi}_{1}(\nabla f(\bar{y}))_{1}=0 \text { and } \tilde{c}_{2}+\tilde{\chi}_{2}(\nabla f(\bar{y}))_{2}=-1 \leq 0 .
$$

Thus, $[N C]$. holds at $\bar{y}=(0,-1)$. 
Remark 2.1. It is worth noting that Theorem 2.1 requires that

$$
\max \left\{\partial^{2} f(z) / \partial x_{i}^{2} \mid z \in \tilde{D}\right\} \leq c_{i},
$$

which itself is a global optimization problem. So, it may not be always easy to find a suitable $c_{i}$ satisfying this condition. However, as we will see later in the Section that this condition can easily be checked for many important classes of problems. On the other hand, it is important to find $c_{i}$ which is as close as $\max \left\{\partial^{2} f(z) / \partial x_{i}^{2} \mid z \in \tilde{D}\right\}$ in order to get an effctive necessary condition. For instance, if we take $c_{2}=0.5$ in Example 2.1, then the point $(0,1)$ also satisfies $[N C]$ and becomes a candidate for a global minimizer.

Corollary 2.1. For $(M P)$, let $d_{i}=\max \left\{\partial^{2} f(z) / \partial x_{i}^{2} \mid z \in \tilde{D}\right\}$, for $i=1,2, \ldots, n$. If $\bar{x} \in D$ is a global minimizer of $(M P)$ then, for each $i=1,2, \ldots, n$,

$$
[N C 1] \quad \frac{1}{2} \quad \tilde{d}_{i}\left(v_{i}-u_{i}\right)+\tilde{\chi}_{i}(\nabla f(\bar{x}))_{i} \leq 0 .
$$

Proof. The conclusion follows from Theorem 2.1 by taking $c_{i}=d_{i}$.

Corollary 2.2. For $(M P)$, let $A=\left(a_{i j}\right) \in S^{n}, a \in \mathbb{R}^{n}$ and let $g$ be a twice continuously differentiable function such that $\left(\partial^{2} g(z) / \partial x_{i}^{2}\right) \geq 0, \forall i=1,2, \ldots, n$ and $\forall z \in \tilde{D}$. Let $f(x)=\frac{1}{2} x^{T} A x+a^{T} x-g(x), x \in \mathbb{R}^{n}$. If $\bar{x} \in D$ is a global minimizer of $(M P)$ then for each $i=1,2, \ldots, n$,

$$
[N C 2] \quad \frac{1}{2} \tilde{a}_{i i}\left(v_{i}-u_{i}\right)+\tilde{\chi}_{i}(a+A \bar{x}-\nabla g(\bar{x}))_{i} \leq 0 .
$$

Proof. Note that

$$
\frac{\partial^{2} f(z)}{\partial x_{i}^{2}}=a_{i i}-\frac{\partial^{2} g(z)}{\partial x_{i}^{2}} \leq a_{i i}, \forall i=1, \ldots, n \text { and } \forall z \in \tilde{D} .
$$

So, [NC2] follows easily from Theorem 2.1 by taking $c_{i}=a_{i i}$.

Corollary 2.3 (Minimization of Difference of Quadratic and Convex Functions). For $(M P)$, let, for each $x \in \mathbb{R}^{n}, f(x)=\frac{1}{2} x^{T} A x+a^{T} x-g(x)$, where $A=\left(a_{i j}\right) \in$ $S^{n}, a \in \mathbb{R}^{n}$ and $g$ is a twice continuously differentiable convex function. If $\bar{x} \in D$ is a global minimizer of $(M P)$ then for each $i=1,2, \ldots, n$,

$$
[N C 3] \quad \frac{1}{2} \tilde{a}_{i i}\left(v_{i}-u_{i}\right)+\tilde{\chi}_{i}(a+A \bar{x}-\nabla g(\bar{x}))_{i} \leq 0 .
$$

Proof. Because $g$ is convex, we have $\left(\partial^{2} g(z) / \partial x_{i}^{2}\right) \geq 0, \forall i \in I$. Hence, [NC3] follows from Corollary 2.2.

Corollary 2.4 (Quadratic Minimization). For $(M P)$, let, for each $x \in \mathbb{R}^{n}, f(x)=$ $\frac{1}{2} x^{T} A x+a^{T} x, A:=\left(a_{i j}\right) \in S^{n}, a \in \mathbb{R}^{n}$. If $\bar{x} \in D$ is a global minimizer of $(M P)$ 
then for each $i=1,2, \ldots n$,

$$
[N C 4] \quad \frac{1}{2} \tilde{a}_{i i}\left(v_{i}-u_{i}\right)+\tilde{\chi}_{i}(a+A \bar{x})_{i} \leq 0 .
$$

Moreover, if $A$ is a diagonal matrix then $\bar{x}$ is a global minimizer of $(M P)$ if and only if $[N C 4]$ holds.

Proof. Clearly $[\mathrm{NC} 4]$ follows from Corollary 2.1 by taking $g=0$. To obtain sufficiency, let $A:=\operatorname{diag}\left(a_{11}, \ldots, a_{n n}\right)$. Then,

$$
f(x)-f(\bar{x})=\frac{1}{2} \sum_{i=1}^{n} a_{i i}\left(x_{i}-\bar{x}_{i}\right)^{2}+\sum_{i=1}^{n}(a+A \bar{x})_{i}\left(x_{i}-\bar{x}_{i}\right) .
$$

The analysis that is similar to that of the proof of Theorem 2.1, shows that $\bar{x}$ is a global minimizer of $(M P)$ if and only if for every $i=1,2, \ldots n$ and $x=$ $\left(x_{1}, \ldots, x_{n}\right)^{T} \in D$

$$
\frac{1}{2} a_{i i}\left(x_{i}-\bar{x}_{i}\right)^{2}+(a+A \bar{x})_{i}\left(x_{i}-\bar{x}_{i}\right) \geq 0
$$

By considering the three cases as in the proof of Theorem 2.1, we see that (8) is equivalent to $[\mathrm{NC} 4]$.

Corollary 2.5 (Minimization of Difference of Convex Functions). For (MP), let $g$ and $h$ be twice continuously differentiable convex functions on $\mathbb{R}^{n}$. Let $f(x)=$ $h(x)-g(x), x \in \mathbb{R}^{n}$. Suppose that, for each $i=1,2, \ldots, n, \max \left\{\partial^{2} h(z) / \partial x_{i}^{2} \mid z \in\right.$ $\tilde{D}\} \leq d_{i}$. If $\bar{x} \in D$ is a global minimizer of $(M P)$ then for each $i=1,2, \ldots n$,

$$
[N C 5] \quad \frac{1}{2} \tilde{d}_{i}\left(v_{i}-u_{i}\right)+\tilde{\chi}_{i}(\nabla h(x)-\nabla g(x))_{i} \leq 0
$$

Proof. Since $g$ is convex, for each $z \in \tilde{D}, \partial^{2} g(z) / \partial x_{i}^{2} \geq 0$. So,

$$
\frac{\partial^{2} f(z)}{\partial x_{i}^{2}}=\frac{\partial^{2} h(z)}{\partial x_{i}^{2}}-\frac{\partial^{2} g(z)}{\partial x_{i}^{2}} \leq d_{i}, \text { for each } z \in \tilde{D} \text {. }
$$

Hence $[N C 5]$ follows from Theorem 2.1 by taking $c_{i}=d_{i}$.

Corollary 2.6. If $\bar{x} \in D$ is a global minimizer of $(M P)$ then, for each $i \in I$,

$$
[N C 6] \quad \tilde{\chi}_{i}(\nabla f(\bar{x}))_{i} \leq 0 .
$$

Proof. The proof is immediate from Theorem 2.1 and so is omitted. 


\section{Minimizing underestimators AND SUfFicient CONDitions}

In this section we obtain sufficient global optimality conditions for the model problem $(M P)$ by underestimation.

Definition (Minimizing Underestimators). A quadratic function $\ell$ on $\mathbb{R}^{n}$ is a minimizing quadratic underestimator of a function $f$ at $\bar{x}$ over $D$ if

$$
\forall x \in D, f(x)-f(\bar{x}) \geq \ell(x)-\ell(\bar{x}) \text { and } \forall x \in D, \ell(x)-\ell(\bar{x}) \geq 0
$$

Clearly, if $f$ has a minimizing quadratic underestimator at $\bar{x}$ then it attains its global minimum at $\bar{x}$.

Lemma 3.1. Let $\bar{x} \in D$ and $Q=\operatorname{diag}\left(q_{1}, q_{2}, \ldots, q_{n}\right) \in S^{n}, q_{i} \in \mathbb{R}, i=$ $1,2, \ldots, n$. If, for each $x \in \tilde{D},\left(\nabla^{2} f(x)-Q\right) \succeq 0$, and

$$
\frac{1}{2} \tilde{q}_{i}\left(v_{i}-u_{i}\right)+\tilde{\chi}_{i}(\nabla f(\bar{x}))_{i} \leq 0, i=1,2, \ldots, n
$$

then $\ell(x)=\frac{1}{2} x^{T} Q x+(\nabla f(\bar{x})-Q \bar{x})^{T} x, x \in \mathbb{R}^{n}$, is a minimizing quadratic underestimator of $f$ at $\bar{x}$ over $D$.

Proof. Let $\phi(x)=f(x)-\ell(x), x \in \tilde{D}$. Then $\nabla \phi(\bar{x})=0$ and $\nabla^{2} \phi(x)=\nabla^{2} f(x)-$ $Q \succeq 0, \forall x \in \tilde{D}$. So $\phi(x)-\phi(\bar{x}) \geq 0$, for all $x \in \tilde{D}$, as $\phi$ is a convex function over $\tilde{D}$. Hence, $f(x)-f(\bar{x}) \geq \ell(x)-\ell(\bar{x}), \forall x \in D$. We now consider

$$
\ell(x)-\ell(\bar{x})=\sum_{i=1}^{n} \frac{q_{i}}{2}\left(x_{i}-\bar{x}_{i}\right)^{2}+\sum_{i=1}^{n}(\nabla f(\bar{x}))_{i}\left(x_{i}-\bar{x}_{i}\right) .
$$

Then, as in the proof of Theorem 2.1, $\ell(x)-\ell(\bar{x}) \geq 0$ for all $x \in D$ if and only if

$$
\frac{1}{2} q_{i}\left(x_{i}-\bar{x}_{i}\right)^{2}+(\nabla f(\bar{x}))_{i}\left(x_{i}-\bar{x}_{i}\right) \geq 0 \quad \text { for each } i=1, \ldots, n
$$

By considering three cases and by using similar analysis as in the proof of Theorem 2.1, we have (9) holds if and only if

$$
\frac{1}{2} \tilde{q}_{i}\left(v_{i}-u_{i}\right)+\tilde{\chi}_{i}(\nabla f(\bar{x}))_{i} \leq 0 \quad \text { for each } i=1,2, \ldots, n
$$

Remark 3.1. The condition, $\left.\nabla^{2} f(x)-Q\right) \succeq 0, \forall x \in \tilde{D}$, means that the function $p$, defined by, $p(x)=f(x)-\frac{1}{2} x^{T} Q x$, is convex over $\tilde{D}$. So, Lemma 3.1 applies to functions $f$ of the form where $f(x)=p(x)+\frac{1}{2} x^{T} Q x$. Hence minimizing quadratic underestimators can easily be found for the sum of a convex and a (not necessarily convex) weighted sum of squares. 
We now see that whenever $\left(\tilde{\chi}_{i}(\nabla f(\bar{x}))_{i}\right) \leq 0, i \in I,(10)$ holds with

$$
q_{i}=\frac{\left.2 \tilde{\chi}_{i}(\nabla f(\bar{x}))_{i}\right)}{v_{i}-u_{i}} .
$$

This leads us to obtain a simple verifiable sufficient condition for global optimality.

Theorem 3.1. For $(M P)$, let $\bar{x} \in D$. Suppose that for each $i \in I, \tilde{\chi}_{i}(\nabla f(\bar{x}))_{i} \leq 0$. If

$$
\begin{array}{r}
\left(\nabla^{2} f(x)+\operatorname{diag}\left(\left(-2 \tilde{\chi}_{1}(\nabla f(\bar{x}))_{1}\right) /\left(v_{1}-u_{1}\right), \ldots,\left(-2 \tilde{\chi}_{n}(\nabla f(\bar{x}))_{n}\right) /\left(v_{n}-u_{n}\right)\right)\right. \\
\forall x, \\
\forall x \in \tilde{D},
\end{array}
$$

then $\bar{x}$ is a global minimizer of $(M P)$.

Proof. Let $Q=\operatorname{diag}\left(q_{1}, q_{2}, \ldots, q_{n}\right)$ and let $\ell(x)=\frac{1}{2} x^{T} Q x+(\nabla f(\bar{x})-Q \bar{x})^{T}, x \in \tilde{D}$. Then $\nabla^{2} f(x)-Q \succeq 0$. Since $\tilde{\chi}_{i}(\nabla f(\bar{x}))_{i} \leq 0, \quad \forall i \in I$, we have $\tilde{q}_{i}=-q_{i}, \quad \forall i \in$ $I \cup J$. Hence

$$
\frac{1}{2} \tilde{q}_{i}\left(v_{i}-u_{i}\right)+\tilde{\chi}_{i}(\nabla f(\bar{x}))_{i} \leq 0 .
$$

Therefore the requirements of Lemma 3.1 are satisfied and hence $\ell$ is a minimizing quadratic underestimator of $f$ at $\bar{x}$. Thus $\bar{x}$ is a global minimizer of $(M P)$.

Example 3.1. Let us consider the problem discussed in Section 2:

$$
\min _{x \in \mathbb{R}^{2}}\left\{x_{1}^{4}+x_{1}^{2}-x_{2}^{2}+x_{2} \mid x_{1} \in[-1,1], x_{2} \in\{-1,1\}\right\} .
$$

Then, $\nabla f(x)=\left(4 x_{1}^{3}+2 x_{1},-2 x_{2}+1\right)^{\mathrm{T}}$ and

$$
\nabla^{2} f(x)=\left(\begin{array}{cc}
12 x_{1}^{2}+2 & 0 \\
0 & -2
\end{array}\right)
$$

Let $\bar{y}=(0,-1)^{\mathrm{T}}$. Note that, for each $x \in D$,

$$
\begin{aligned}
\nabla^{2} f(x)-\operatorname{diag}\left(\tilde{\chi}_{i}(\nabla f(\bar{y}))_{i}\right)=\left(\begin{array}{cc}
12 x_{1}^{2}+2 & 0 \\
0 & -2
\end{array}\right)-\left(\begin{array}{cc}
0 & 0 \\
0 & -3
\end{array}\right) \\
=\left(\begin{array}{cc}
12 x_{1}^{2}+2 & 0 \\
0 & 1
\end{array}\right) \succeq 0 .
\end{aligned}
$$

Moreover, $\tilde{\chi}_{1}(\nabla f(\bar{y}))_{1}=\tilde{\chi}_{2}(\nabla f(\bar{y}))_{2}=0$. Thus, $\bar{y}=(0,-1)$ is a global minimizer of the problem.

Corollary 3.1. Let $g$ be a twice continuously differentiable convex function on $\mathbb{R}^{n}, A \in S^{n}$ and $a \in R^{n}$. Let $f(x)=g(x)-\frac{1}{2} x^{\mathrm{T}} A x+a^{\mathrm{T}} x, x \in \mathbb{R}^{n}$. Suppose that for each $i \in I, \tilde{\chi}_{i}(\nabla(g(\bar{x}))-A \bar{x}+a)_{i} \leq 0$. If

$$
\begin{aligned}
\left(\operatorname { d i a g } \left(\left(-2 \tilde{\chi}_{1}(\nabla g(\bar{x})-A \bar{x}+a)_{1}\right) /\right.\right. & \left.\left(v_{1}-u_{1}\right)\right), \ldots, \\
& \left.\left.\left(-2 \tilde{\chi}_{n}(\nabla g(\bar{x})-A \bar{x}+a)_{n}\right) /\left(v_{n}-u_{n}\right)\right)-A\right) \succeq 0
\end{aligned}
$$


for all $x \in \tilde{D}$ then $\bar{x}$ is a global minimizer of $(M P)$.

Proof. Since $g$ is convex, $\nabla^{2} g(x) \succeq 0, \forall x \in \tilde{D}$. Hence $\nabla^{2} f(x)+\operatorname{diag}\left(\left(-2 \tilde{\chi}_{1}(\nabla g(\bar{x})-\right.\right.$ $\left.\left.A \bar{x}+a)_{1}\right) /\left(v_{1}-u_{1}\right), \ldots,\left(-2 \tilde{\chi}_{n}(\nabla g(\bar{x})-A \bar{x}+a)_{n}\right) /\left(v_{n}-u_{n}\right)\right)=\nabla^{2} g(x)+(-A-$ $\left.\operatorname{diag}\left(\left(2 \tilde{\chi}_{1}(\nabla g(\bar{x})-A \bar{x}+a)_{1}\right) /\left(v_{1}-u_{1}\right), \ldots,\left(2 \tilde{\chi}_{n}(\nabla g(\bar{x})-A \bar{x}+a)_{n}\right) /\left(v_{n}-u_{n}\right)\right)\right) \succeq 0$. So, the conclusion follows from Theorem 3.1.

Finally, we observe from Theorem 3.1 that, for $(M P)$, if $f(x)=\frac{1}{2} x^{\mathrm{T}} A x+a^{\mathrm{T}} x$, $x \in \mathbb{R}^{n}$, and if $\left.A+\operatorname{diag}\left(\left(-2 \tilde{\chi}_{1}(A \bar{x}+a)\right)_{1}\right) /\left(v_{1}-u_{1}\right), \ldots,\left(-2 \tilde{\chi}_{n}(A \bar{x}+a)\right)_{n}\right) /\left(v_{n}-\right.$ $\left.\left.u_{n}\right)\right) \succeq 0$ and $\tilde{\chi}_{i}(A \bar{x}+a)_{i} \leq 0, i \in I$ then $\bar{x} \in D$ is a global minimizer of $(M P)$.

\section{REFERENCES}

[1] A. Beck and M. Teboulle, Global optimality conditions for quadratic optimization problems with binary constraints. SIAM J. Optim. 11 (2000) 179-188.

[2] E. Cela, The quadratic assignment problem: theory and algorithms. Kluwer Academic Publishers (1998).

[3] P. De Angelis, P. Pardalos and G. Toraldo, Quadratic programming with box constraints, in Developments in global optimization, edited by Bomze I. et al. Kluwer Acad. Publ., Dordrecht (1997) 73-93.

[4] C.A. Floudas and P.M. Pardalos, Optimization in computational chemistry and molecular biology: Local and global approaches. Kluwer Academic Publishers (2000).

[5] N.Q. Huy, V. Jeyakumar and G.M. Lee, Sufficient global optimality conditions for multiextremal smooth minimization problems with bounds and linear matrix inequality constraints, The ANZIAM J. 47 (2006) 439-450.

[6] N.Q. Huy, V. Jeyakumar and G.M. Lee, Globally minimizing smooth functions with simple constraints: Necessary, and sufficient optimality conditions (submitted).

[7] V. Jeyakumar and N.Q. Huy, Global minimization of difference of quadratic and convex functions over box or binary constraints, To appear in Optimization Letters, DOI: 10.1007/s11590-007-0053-6 (2008).

[8] V. Jeyakumar, A.M. Rubinov and Z.Y. Wu, Sufficient global optimality conditions for nonconvex quadratic minimization problems with box constraints. J. Global Optim. 36 (2006) 461-468.

[9] V. Jeyakumar, A.M. Rubinov and Z.Y. Wu, Nonconvex quadratic minimization with quadratic constraints: Global optimality conditions. Math. Program. 110 (2007) 521-541.

[10] M. Junger, A. Martin, G. Reinelt and R. Weismantel, 0/1 optimization and a decomposition approach for the placement of electronic circuits. Math. Program. 63 (1994) 257-279.

[11] R.F. Marcia, J.C. Mitchell and J.B. Rosen, Iterative convex quadratic approximation for global optimization in protein docking, Comput. Optim. Appl. 32 (2005) 285-297.

[12] P.M. Pardalos and G.P. Rodgers, Computational aspects of quadratic zero-one programming, Computing 45 (1990) 131-144.

[13] M.C. Pinar, Sufficient global optimality conditions for bivalent quadratic optimization, $J$. Optim. Theor. Appl. 122 (2004) 433-440.

[14] M.C. Pinar and M. Teboulle, On semidefinite bounds for maximization of a non-convex quadratic objective over $l_{1}$ unit ball. RAIRO Oper. Res. 40 (2006) 253-265.

[15] Z. Wu, V. Jeyakumar, A.M. Rubinov, Sufficient conditions for global optimality of bivalent nonconvex quadratic programs with inequality constraints. J. Optim. Theor. Appl. 133 (2007) 123-130. 